\title{
Regularized quadrature and phase tracking from a single closed-fringe interferogram
}

\author{
Manuel Servin \\ Centro de Investigaciones en Optica A.C., Apartado Postal 1-948, 37150 Leon, Guanajuato, Mexico \\ Jose Luis Marroquin \\ Centro de Investigaciones en Matematicas A.C., Apartado Postal 402, 36000 Guanajuato, Guanajuato, Mexico \\ Juan Antonio Quiroga \\ Departamento de Optica, Universidad Complutense de Madrid, Ciudad Universitaria S/N, 2841 Madrid, Spain \\ Received August 4, 2003; revised manuscript received November 12, 2003; accepted November 20, 2003 \\ A new sequential phase demodulator based on a regularized quadrature and phase tracker system (RQPT) is \\ applied to demodulate two-dimensional fringe patterns. This RQPT system tracks the fringe pattern's \\ quadrature and phase in a sequential way by following the path of the fringes. To make the RQPT system \\ more robust to noise, the modulating phase around a small neighborhood is modeled as a plane and the \\ quadrature of the signal is estimated simultaneously with the fringe's modulating phase. By sequentially \\ calculating the quadrature of the fringe pattern, one obtains a more robust sequential demodulator than was \\ previously possible. This system may be applied to the demodulation of a single interferogram having closed \\ fringes. (C) 2004 Optical Society of America
}

OCIS codes: $100.5070,100.2650,120.5050,120.3180$.

\section{INTRODUCTION}

Most experimental data obtained by using full-field optical metrology are encoded as a wave front (phase) that modulates the fringes of an interferometric image. ${ }^{1}$ The aim of fringe analysis is to estimate the modulating twodimensional (2D) phase of these fringe patterns. When a linear spatial phase with a large slope (a carrier) is added to the wave front under analysis, one obtains a spatialcarrier-frequency interferogram. When the phase of interest is smooth and a linear carrier is added, the fringe pattern can be easily demodulated by using wellunderstood and widely used spatial carrier interferometry techniques. ${ }^{2}$

On the other hand, if the experiment at hand permits one to obtain several interferograms over a period of time, one may introduce a temporal carrier into the modulating phase. $^{3}$ In this case one varies the modulating phase by using a linear temporal carrier, so every interferogram will have a predefined piston phase difference. Then, using several phase-stepped interferograms, one may easily obtain the modulating phase.

Sometimes, however, the very nature of the experimental setup may not allow one to take one or several interferometric images having a spatial and/or temporal carrier. ${ }^{4}$ These cases frequently arise in the analysis of fast transient phenomena, where it is difficult or impossible to introduce a spatial or temporal carrier. In these cases we have no choice but to deal with a single or a series of interferograms without a carrier, possibly containing closed fringes, where the phase variation is not a monotonic function of space or time. In this situation it is impossible to separate the information of interest with a linear filter. However, there are still some possible ways to deal with these nonmonotonic modulating phase interferograms. One recent method was proposed by Larkin et al., ${ }^{5}$ and, more recently, another, closely related to that one, was proposed by Servin et al. ${ }^{6}$ In these two cases the phase estimation problem is factored into two operators. One is an isotropic 2D Hilbert transform, and the other is the orientation $2 \pi$ of the fringes. The orientation of the fringes is the more difficult step and must be done in a sequential way. ${ }^{7}$ The regularized quadrature and phase tracker (RQPT) system presented here uses, instead, a single system that is capable of demodulating in a robust way a single interferogram containing closed fringes.

The RQPT presented in this work may be considered a significant improvement on a previously published phase demodulation system called the regularized phase tracker (RPT). ${ }^{8}$ The improvement resides in the sequential quadrature estimation of the interferogram's fringes by the RQPT. Although the main objective of any fringe pattern demodulation technique is to find the modulating phase, it is of interest to note (as we will see) that the sequential calculation of the interferogram's quadrature highly improves the robustness of the RPT system presented in past publications. So this new RQPT sequentially calculates the quadrature of the fringe pattern as a by-product to obtain a phase-tracking scheme that is more robust than the previous RPT demodulation algorithm.

The presentation plan for the paper is the following: 
In Subsection 2.A we review the phase-locked loop (PLL) ${ }^{9-11}$ which is the first one-dimensional (1D) sequential phase demodulator used to analyze 2D interferograms, because an important idea that is used in the RQPT is drawn directly from the PLL system. The motivation for a nonregularized quadrature and phase tracker (QPT) demodulation system in one dimension is presented in Subsection 2.B. In Section 3 this $1 \mathrm{D}$ nonregularized (QPT) system is generalized to two dimensions. In Section 4 we regularize the QPT to obtain the sought-after $2 \mathrm{D}$ regularized QPT (RQPT). We continue to Section 5, where we demodulate an experimentally obtained electronic speckle interferogram and then a complicated, noisy, computer-generated fringe pattern. In Section 6 some conclusions of the main results of the paper are given.

\section{SEQUENTIAL PHASE DEMODULATING SYSTEMS}

In this section we present the motivation for a $1 \mathrm{D}$ nonregularized QPT demodulation system. We begin by describing the standard model for a $2 \mathrm{D}$ fringe pattern as obtained by an optical interferometer:

$$
I(\mathbf{r})=a(\mathbf{r})+b(\mathbf{r}) \cos [\phi(\mathbf{r})],
$$

where $\mathbf{r}=(x, y)$ represents a point in $2 \mathrm{D}$ space. The smooth function $a(\mathbf{r})$ is a low-frequency signal and represents the background illumination. The function $b(\mathbf{r})$ is also a low-frequency signal, which represents the $2 \mathrm{D}$ contrast variation of the fringe pattern. The signal $\phi(\mathbf{r})$ is the information to be recovered, which is related to the physical magnitude under measurement. Throughout this work we will assume that the modulating phase $\phi(\mathbf{r})$ is continuous and smooth.

Let us start by analyzing the PLL, which was the first phase-tracking system that was applied to fringe pattern demodulation. ${ }^{9}$ A brief review of the PLL is convenient because the QPT presented here uses a fundamental idea that is drawn directly from the PLL system.

\section{A. Phase-Locked Loop System}

As we will see, both the PLL and RQPT systems are capable of demodulating only open-fringe patterns if no attention is paid to the $2 \mathrm{D}$ sequential scanning strategy. Let us start with an analysis of a 1D carrier frequency signal and how the PLL and RQPT systems presented in this work demodulate it. The standard mathematical model for a 1D fringe pattern with a linear carrier is the following:

$$
I_{1}(x)=a(x)+b(x) \cos \left[\omega_{0} x+\phi(x)\right],
$$

where the carrier frequency $\omega_{0}$ must be greater than the maximum frequency content of the modulating phase $\phi(x)$, or $\omega_{0}>|\partial \phi(x) / \partial x|$ for all $x$. The PLL (as well as the RQPT) works best when the background signal is removed or is highly attenuated. Then, using a high-pass filter, one is able to rewrite Eq. (2) as

$$
I(x)=b(x) \cos \left[\omega_{0} x+\phi(x)\right] .
$$

The continuous first-order PLL system is usually described by the following nonlinear dynamic system:

$$
\frac{\mathrm{d} \hat{\phi}(x)}{\mathrm{d} x}=\tau I(x) \sin \left[\omega_{0} x+\hat{\phi}(x)\right]
$$

where $\hat{\phi}(x)$ denotes the estimated modulating phase and $\tau$ is a constant related to the bandpass of the PLL. To understand the basic functioning of this system, we rewrite the last equation in integral form as

$$
\hat{\phi}(x)=\tau \int_{-\infty}^{x} \cos \left[\omega_{0} \xi+\phi(\xi)\right] \sin \left[\omega_{0} \xi+\hat{\phi}(\xi)\right] \mathrm{d} \xi,
$$

where we have assumed that we have $b(x) \approx 1.0$. This equation may be rewritten as

$$
\begin{aligned}
\hat{\phi}(x)= & \frac{\tau}{2} \int_{-\infty}^{x}\left\{\sin [\phi(\xi)-\hat{\phi}(\xi)]+\sin \left[2 \omega_{0} \xi+\hat{\phi}(\xi)\right.\right. \\
& +\phi(\xi)]\} \mathrm{d} \xi
\end{aligned}
$$

As we can see from this equation, there are two terms: The first varies slowly because the modulating phase $\phi(x)$ is a continuous smooth function, while the second varies twice as fast as the original fringes. Because the integral is a first-order low-pass filter, the fast-varying term is highly reduced and may be neglected. Therefore one may rewrite, for analysis purposes, a simplified version of the PLL dynamic system as

$$
\frac{\mathrm{d} \hat{\phi}(x)}{\mathrm{d} x}=\tau \sin [\phi(x)-\hat{\phi}(x)] .
$$

When the PLL is operating in lock, the estimated phase $\hat{\phi}(x)$ follows the modulating phase $\phi(x)$ very closely, so the difference $\phi(x)-\hat{\phi}(x)$ is small and the sine function may be approximated by its argument. Doing this, we finally arrive at

$$
\frac{\mathrm{d} \hat{\phi}(x)}{\mathrm{d} x}=\tau[\phi(x)-\hat{\phi}(x)] .
$$

This last approximation permits one to understand why the demodulated phase $\hat{\phi}(x)$ obtained with a first-order PLL follows closely the modulating phase of the fringes, $\phi(x)$.

Finally, let us write the spatially discretized (or digital) version of the PLL system that is obtained by discretizing the spatial coordinate $x$. Using first-order differences to approximate the continuous phase derivative, one obtains a digital first-order PLL as

$$
\hat{\phi}(x+1)=\hat{\phi}(x)+\tau I(x) \sin \left[\omega_{0} x+\hat{\phi}(x)\right] .
$$

With this equation one sees that the currently evaluated phase $\hat{\phi}(x+1)$ at the site $x+1$ uses the previously estimated phase $\hat{\phi}(x)$ as a predictor with an update given by $\tau I(x) \sin [\omega x+\hat{\phi}(x)]$, which corrects the preceding estimate by a small amount. Finally, let us mention that the phase estimated by the PLL is already unwrapped, so that no additional unwrapping system is required to obtain the desired smooth modulating phase.

We have seen that the PLL system is capable of demodulating a carrier frequency signal whenever we have an estimate of the carrier frequency $\omega_{0}$ and the two terms in Eq. (6) are well separated in frequency space. If the 
spectral distance between these two terms is not large enough, they overlap and the higher-frequency carrier will appear as an artifact in the estimated phase. This situation arises if a low-frequency carrier is modulated by a wideband phase, and in this case it is not possible to use the PLL system to demodulate these fringes. We will see in Subsection 2.B the new phase-tracking demodulator (QPT) that overcomes these difficulties.

\section{B. Quadrature and Phase Tracker Estimator System}

Let us start by intuitively motivating how one can arrive at another phase-tracking scheme that overcomes the PLL system limitations. In this case, instead of starting with a dynamic system and then explaining why it works, as in the case of the PLL, we will proceed by postulating a cost functional operating over the estimated phase space $\hat{\phi}(x)$, hoping that the solution $\hat{\phi}(x)$ that renders this functional a minimum is the expected demodulated phase.

For this let us naively postulate a simple quadratic cost functional that we know the searched-for solution will render a minimum. This cost functional is

$$
U=\{I(x)-\cos [\hat{\phi}(x)]\}^{2},
$$

where we have simplified our 1D fringe pattern model by assuming that $a(x) \approx 0$ and $b(x) \approx 1$. The optimum function is obtained by the signal

$$
\hat{\phi}(x)=\arccos [I(x)] ;
$$

such a phase is illustrated in Fig. 1(a), and it is clear that this is not what we were looking for. Then, let us continue working over the same idea by introducing another term to our cost functional that imposes an additional constraint on $\hat{\phi}(x)$ by requiring that one also approximate the quadrature of the fringes. The quadrature of $I(x)$ $=\cos [\phi(x)]$ is $\sin [\phi(x)]=-I_{x}(x) / \phi_{x}(x)=-I_{x}(x) / \omega(x)$, where $\omega(x)$ is the local frequency. With this addition our new cost functional now reads

$$
U=\{I(x)-\cos [\hat{\phi}(x)]\}^{2}+\left\{I_{x}(x)+\hat{\omega}(x) \sin [\hat{\phi}(x)]\right\}^{2},
$$

where the new unknown function $\hat{\omega}(x)$ is the derivative of $\hat{\phi}(x)$ with respect to $x$ and $I_{x}(x)$ is approximated by firstorder differences as $I(x)-I(x-1)$ in our discrete $1 \mathrm{D}$ space. Now the optimum for $\hat{\omega}(x)$ and $\hat{\phi}(x)$ is obtained, and the solution looks like Fig. 1(b). This solution was found iteratively by tacking small steps along the direction of steepest descent of $U$, i.e., along the direction of minus the gradient of $U$ with respect to the optimizing variables. That is,

$$
\begin{aligned}
& \hat{\phi}(x)^{k+1}=\hat{\phi}(x)^{k}-\mu \frac{\partial U}{\partial \hat{\phi}(x)}, \\
& \hat{\omega}(x)^{k+1}=\hat{\omega}(x)^{k}-\mu \frac{\partial U}{\partial \hat{\omega}(x)},
\end{aligned}
$$

where $\mu$ is a fixed step size. If one uses "natural" [given that we do not know anything about $\phi(x)$ or $\hat{\omega}(x)]$ zero initial conditions

$$
\hat{\phi}(x)^{0}=0, \quad \hat{\omega}(x)^{0}=0,
$$

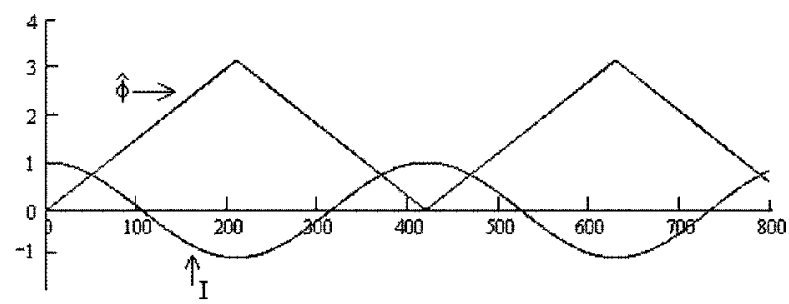

(a)

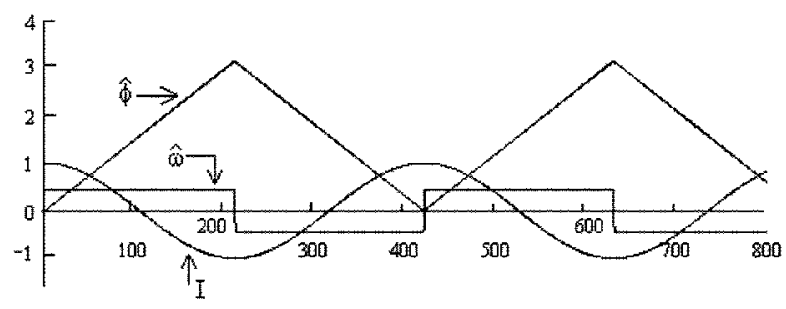

(b)

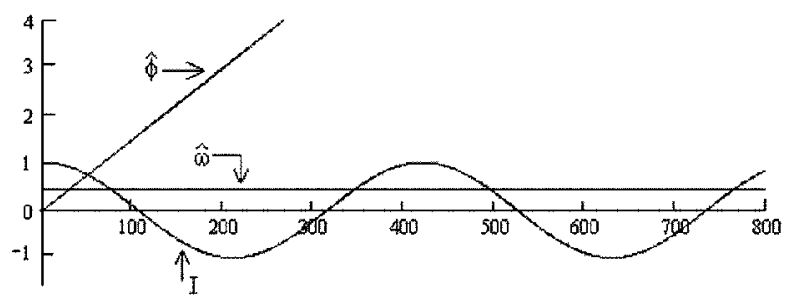

(c)

Fig. 1. Phase demodulation of a sinusoidal signal. (a) Estimated phase given by $\hat{\phi}=\cos ^{-1}(\phi)$, which is the minimum of $U$ in Eq. (10); (b) minimizing function of Eq. (12) along with zero initial conditions [Eqs. (15)]; (c) function that minimizes Eq. (12) but now using as initial conditions the previously found estimates [Eqs. (16)].

one obtains the estimated phase and local frequency shown in Fig. 1(b). It is apparent that we have made no progress toward the desired solution: We just found what we already knew from our first attempt. The reason is that although we have added another datum, which is $I_{x}(x)$, we also have added a new unknown, which is $\hat{\omega}(x)$, so we have returned to our starting point.

If, however, we could have a rough estimate for the newly created unknown $\hat{\omega}(x)$, introducing this value into our last cost functional, we would be closer to knowing the quadrature of the fringes at site $x$. One way to obtain this rough estimate is by using the same PLL trick, namely, to use the previously found estimate [in this case, for $\hat{\omega}(x)$ ] at the already visited site $x-1$ as our initial guess. Using as initial estimate $\hat{\omega}(x)^{0}=\hat{\omega}(x-1)^{\infty}$ in Eq. (14), one gets a better estimate for the desired $\hat{\phi}(x)$ than was previously possible. Once a better $\hat{\phi}(x)$ is found, we then use it to improve our estimate for the actual $\hat{\omega}(x)$, which in turn is used to improve our $\hat{\phi}(x)$. We may continue this iterative process until we find both unknowns within a certain predefined error. By doing this, one obtains the desired (wrapped) demodulated phase. Of course, the very first site being demodulated within the fringe pattern will not have a previously found esti- 
mation; for that unique seed site, the initial conditions for $\hat{\phi}(x)$ and $\hat{\omega}(x)$ may be set to zero.

An alternative explanation that supports the use of the stable estimated $\hat{\omega}(x-1)^{\infty}$ as initial condition for $\hat{\omega}(x)^{0}$ may be found by observing the unwanted behavior that the local frequency $\hat{\omega}(x)$ has when zero initial conditions are chosen for Eq. (14) [Fig. 1(b)]. The desired estimated frequency $\hat{\omega}(x)$ should be a constant and not a square function. The discontinuities of $\hat{\omega}(x)$ are due to the fact that the driving term $\partial U / \partial \hat{\omega}(x)$ [in Eq. (14)] has a very low value in the neighborhood of the extrema of the fringe pattern. In these places, only the driving term for $\hat{\phi}(x)$, which is $\partial U / \partial \hat{\phi}(x)$, has a significant value and pulls down the estimated phase in the absence of a driving force for the instantaneous frequency $\hat{\omega}(x)$. So $\hat{\omega}(x)$ does not have any choice but to follow the changes commanded by the estimated phase $\hat{\phi}(x)$, which finally switches $\hat{\omega}(x)$ to a constant negative value. To get out of this situation, one should use as initial condition for $\hat{\omega}(x)^{0}$ [in Eq. (14)] the stable value for $\hat{\omega}(x-1)^{\infty}$ found at the previously visited site $x-1$. In this way, instead of having a negligible value for $\hat{\omega}(x)$, one will have as initial estimate the value $\hat{\omega}(x-1)^{\infty}$, which is a significant positive value. In that case the natural solution for the estimated phase at $x$ is to continue its monotonically increasing behavior [see Fig. 1(c)]. Once this critical point has been passed, the driving term $\partial U / \partial \hat{\omega}(x)$ will have again a high and welldetermined value, forcing the frequency $\hat{\omega}(x)$ to remain positive until the next critical point of the fringe pattern appears.

We may also use the previous estimation at $\hat{\phi}(x-1)$ as initial guess for $\hat{\phi}(x)$, as is done in the PLL. This process of using the two previously found estimates for $\phi(x)$ and $\hat{\omega}(x)$ permits us not only to find the sought-after phase and frequency at $x$ but also to unwrap the phase being demodulated, as in the PLL case. In summary, the sequential QPT system is still given by Eqs. (13) and (14), but now the initial conditions to be used are

$$
\hat{\phi}(x)^{0}=\hat{\phi}(x-1)^{\infty}, \quad \hat{\omega}(x)^{0}=\hat{\omega}(x-1)^{\infty}
$$

where $\hat{\omega}(x-1)^{\infty}$ and $\hat{\phi}(x-1)^{\infty}$ denote the stable point $(\hat{\phi}, \hat{\omega})$ of the previously demodulated site at $x-1$. Using these initial conditions, one now obtains the expected continuous phase. Summarizing, the QPT system has two important advantages over the PLL: First, no carrier frequency estimate is needed, and second, one does not need to worry about the risk of having overlapping spectra, unlike the case for the PLL system.

\section{DEMODULATION OF TWO-DIMENSIONAL CLOSED-FRINGE PATTERNS USING THE SEQUENTIAL QUADRATURE AND PHASE TRACKER ESTIMATOR}

Before further discussion let us generalize our 1D-QPT to two dimensions. This generalization is straightforward and is obtained by minimization of the following cost functional:

$$
\begin{aligned}
U=[ & I-\cos (\hat{\phi})]^{2}+\left[I_{x}+\hat{\omega}_{x} \sin (\hat{\phi})\right]^{2}+\left[I_{y}\right. \\
& \left.+\hat{\omega}_{y} \sin (\hat{\phi})\right]^{2},
\end{aligned}
$$

where the spatial position dependence $\mathbf{r}=(x, y)$ of $I_{x}$, $I_{y}, \hat{\phi}, \hat{\omega}_{x}$, and $\hat{\omega}_{y}$ was omitted for clarity purposes. Now we need to optimize for three functions, namely, $\hat{\phi}, \hat{\omega}_{x}$, and $\hat{\omega}_{y}$. The optimizing system at the site $\mathbf{r}_{i}$ has a similar form to that of the 1D-QPT system; in this case we have

$$
\begin{gathered}
\hat{\phi}\left(\mathbf{r}_{i}\right)^{k+1}=\hat{\phi}\left(\mathbf{r}_{i}\right)^{k}-\mu \frac{\partial U}{\partial \hat{\phi}\left(\mathbf{r}_{i}\right)}, \\
\hat{\omega}_{x}\left(\mathbf{r}_{i}\right)^{k+1}=\hat{\omega}_{x}\left(\mathbf{r}_{i}\right)^{k}-\mu \frac{\partial U}{\partial \hat{\omega}_{x}\left(\mathbf{r}_{i}\right)}, \\
\hat{\omega}_{y}\left(\mathbf{r}_{i}\right)^{k+1}=\hat{\omega}_{y}\left(\mathbf{r}_{i}\right)^{k}-\mu \frac{\partial U}{\partial \hat{\omega}_{y}\left(\mathbf{r}_{i}\right)},
\end{gathered}
$$

with initial conditions given by

$$
\begin{aligned}
\hat{\phi}\left(\mathbf{r}_{i}\right)^{0} & =\hat{\phi}\left(\mathbf{r}_{i-1}\right)^{\infty}, \quad \hat{\omega}_{x}\left(\mathbf{r}_{i}\right)^{0}=\hat{\omega}_{x}\left(\mathbf{r}_{i-1}\right)^{\infty}, \\
\hat{\omega}_{y}\left(\mathbf{r}_{i}\right)^{0} & =\hat{\omega}_{y}\left(\mathbf{r}_{i-1}\right)^{\infty},
\end{aligned}
$$

where $\mathbf{r}_{i}$ is the current $i$ site under optimization and $\mathbf{r}_{i-1}$ is the previous one. In the $2 \mathrm{D}-\mathrm{QPT}$ case the $2 \mathrm{D}$ fringe signal can be demodulated whenever

$$
\|\hat{\omega}(\mathbf{r})\|=\left[\hat{\omega}_{x}^{2}(\mathbf{r})+\hat{\omega}_{y}^{2}(\mathbf{r})\right]^{1 / 2}>0 ;
$$

according to the last expression, the 2D-PT system will successfully demodulate open low-frequency fringes regardless of their $2 \mathrm{D}$ orientation.

We have seen that the QPT system can demodulate carrier frequency fringe patterns without the need for an explicit linear carrier. As we have also seen, this has the advantage of demodulating very-low-frequency fringes without the worry about higher-frequency cross-tacking signals that occurs in the PLL case. But how can this 2D-QPT system be used to demodulate closed-fringe interferograms where the spatial phase variation is nonmonotonic, since the 2D-QPT demodulates only monotonically increasing phase? The answer is by following the path of the fringes, that is, not by using a row-by-row scanning strategy (as in a television set) but by following the path traced by the fringes themselves. One way of achieving this is to use an algorithm published in Ref. 12 that employs the concept of signal "quality." This scanning strategy was originally used to sequentially unwrap noisy phase maps. This algorithm first classifies regions of the image according to how good the signal-to-noise ratio is around a given neighborhood. In that case ${ }^{12}$ one starts the sequential unwrapping algorithm by unwrapping first the best data (less noisy) and afterward the noisier image regions.

In our 2D-QPT case we are not classifying our data as a function of its signal-to-noise ratio. We simply decide in an arbitrary way that our data will have two qualities, which are

$$
\begin{aligned}
& \text { if } I(x)>0, \text { good data, } \\
& \text { if } I(x) \leqslant 0, \text { bad data. }
\end{aligned}
$$


This is shown, for example, in Figs. 3(a) and 3(b) below. Using this arbitrary classification (actually the negative of the above statement could also have been used) coupled with the quality-following scanning strategy, ${ }^{12}$ one is able preferably to follow a scanning path defined by the fringes. ${ }^{8}$ The main and essential advantage of following the fringes is to avoid crossing straight through the critical points of the modulating phase. The reason is that the 2D-QPT system does not estimate the local curvature of the modulating phase (only its value and gradient), so the 2D-QPT does not know how to handle the variety of critical points (minima, maxima, or saddles). In contrast, if the 2D-QPT system continually follows the fringe path, it always sees open fringes all over the $2 \mathrm{D}$ interferogram. In other words, a scan of the interferogram along the fringe paths behaves roughly like a coordinate transformation where closed fringes are transformed into open fringes. Therefore whenever the 2D-QPT system encircles these critical points, the 2D-QPT system will never know that it was actually demodulating a closed-fringe interferogram. Eventually, after demodulating the neighborhood (at a small distance) of these critical points, the 2D-QPT will finally have to deal with them. In those critical regions the local gradient $\|\hat{\omega}(\mathbf{r})\|$ is almost zero, so the only remaining term in the functional is the first one. However delicate, in this final step of the demodulation process the problem is much less severe given that we have already demodulated the phase surrounding these critical points, and the solution will grow toward the remaining undemodulated region in a robust way.

This fringe-following 2D-QPT algorithm makes use of the local geometry of the phase being recovered by modeling the local phase $\hat{\phi}(\mathbf{r})$ by a small one-pixel plane determined by the triad $\hat{\phi}, \hat{\omega}_{x}, \hat{\omega}_{y}$. Additionally, the fringe-following 2D-QPT also has global information about the phase at its disposal and uses it by scanning the interferogram following its fringes.

Figure 2 shows some snapshots of the QPT demodulating process. We can see how the $2 \mathrm{D}$ fringe pattern is being phase demodulated following the fringe scanning strategy. As can also be seen, the critical points are not processed until its surrounding phase is already demodulated. In this case we have obtained the path-following data [Fig. 2(b)] by splitting the fringe's gray-level range into four regions instead of just two as in expressions (23) and (24).

\section{REGULARIZING THE QUADRATURE AND PHASE TRACKER ESTIMATOR}

In Section 3 we have shown how the sequential QPT may be used to demodulate closed-fringe interferograms modulated by a smooth continuous function. However, the QPT system just presented is not at all robust with respect to noise. To improve the QPT robustness, we need to regularize the cost functional. In classical regularization one normally introduces a smoothing term into the cost functional. The smoothing term is normally built by using integrals of derivative operators applied to the field that one wants to recover. ${ }^{13}$ This is the standard way of regularizing an inverse linear problem when the transforming linear operator is not invertible or is ill

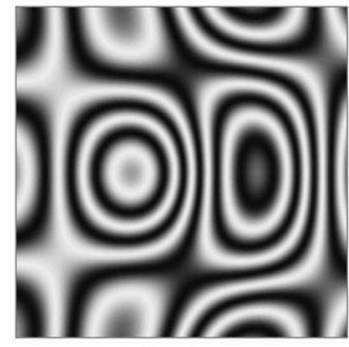

(a)

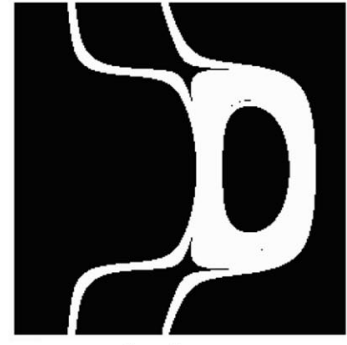

(c)

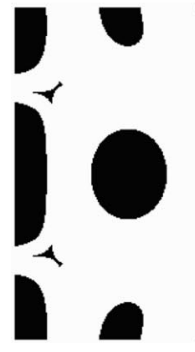

(e)

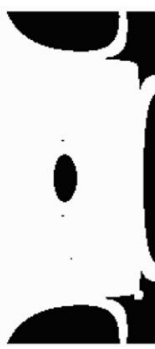

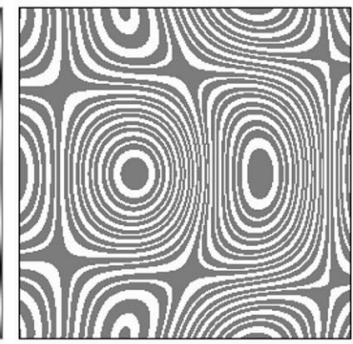

( b )

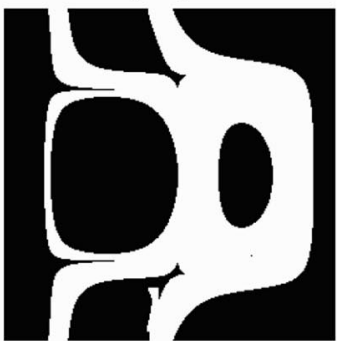

(d)

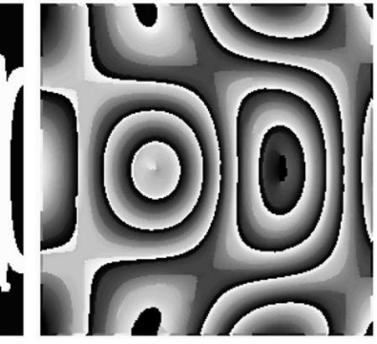

(f)
Fig. 2. Demodulation of a 2D fringe pattern using the nonregularized quadrature and phase tracker (QPT) estimator. (a) Given noiseless computer-generated fringe pattern. (b) Path followed by the sequential demodulating system. Whiter paths are preferably followed as being the sites with "higher quality." (c)(e) Path demodulation progress of the QPT system. (f) Demodulated phase. Although the QPT demodulator finds the phase unwrapped, the phase was rewrapped to compare it with (a).

conditioned. Another characteristic of classical regularization algorithms is that the functional used to find the inverse field is optimized globally at each iteration. That is, one updates all the sites within the region $\Omega$ where one wants to recover the inverse field at each global iteration. In contrast, in the QPT case, one finds the modulating phase and the fringe's quadrature sequentially. So one cannot use differential operators to regularize the QPT functional. Fortunately, one can find a way to regularize this functional by assuming that within a neighborhood $\left(N_{x, y}\right)$ around the point $(x, y)$, one may model the modulating phase as a plane. So one takes as a parametric model for the local phase in the neighborhood of $(x, y)$ the plane given by

$$
\begin{aligned}
p(\epsilon, \eta)= & \hat{\phi}(x, y)+\hat{\omega}_{x}(x, y)(x-\epsilon) \\
& +\hat{\omega}_{y}(x, y)(y-\eta) .
\end{aligned}
$$

With this plane model, the local functional looks like

$$
\begin{aligned}
U= & \sum_{(\epsilon, \eta) \in N_{x, y}}\left\{[I-\cos (p)]^{2}+\left[I_{x}+\hat{\omega}_{x} \sin (p)\right]^{2}\right. \\
& \left.+\left[I_{y}+\hat{\omega}_{y} \sin (p)\right]^{2}\right\}
\end{aligned}
$$


where we have omitted the $(x, y, \epsilon, \eta)$ dependence for clarity purposes. Now the optimization proceeds by taking into account not only the current site $(x, y)$ but a $2 \mathrm{D}$ neighborhood $N_{x, y}$ around it. The size of the neighborhood normally used varies from a $3 \times 3$ up to an 11 $\times 11$ pixel window or more depending on the signal-tonoise ratio of the interferogram. The larger the size of the fitting plane $p(\epsilon, \eta)$, the better the noise rejection obtained. Of course, the size of the fitting plane is limited by how reasonable it is to consider the interferogram's phase within the neighborhood $(\epsilon, \eta) \in N_{x, y}$ as a plane. Finally, one may still increase a little bit more the RQPT noise robustness by adding another term that measures the distance between the regularizing plane $p(x, y)$ and previously estimated values of demodulated phase $\hat{\phi}(\epsilon, \eta)$ within $N_{x, y}$. With this last addition the nonlinear cost functional is

$$
\begin{aligned}
U= & \sum_{(\epsilon, \eta) \in N_{x, y}}\left\{[I-\cos (p)]^{2}+\left[I_{x}+\hat{\omega}_{x} \sin (p)\right]^{2}\right. \\
& \left.+\left[I_{y}+\hat{\omega}_{y} \sin (p)\right]^{2}+\lambda(\hat{\phi}-p)^{2} m\right\},
\end{aligned}
$$

where the function $m(\epsilon, \eta)$ is an indicator function that has a value of 1 when the site at $(\epsilon, \eta)$ has already been estimated and of 0 otherwise. The parameter $\lambda$ is a regularization parameter that controls, along with the size of the regularizing plane, the highest-frequency content of the demodulated signal $\hat{\phi}(x, y)$. The value of the $\lambda$ parameter is not very critical; for example, all numerical experiments that we have made use $\lambda=5$ with good results.

\section{EXPERIMENTAL AND SIMULATION RESULTS}

We show in Fig. 3 the application of the RQPT system for demodulating an experimentally obtained speckle fringe pattern. Again, we show in this figure some snapshots in the demodulating process to see how the sequential strategy along the fringes is performed. Although the noise in this fringe pattern is moderately high, the fringe pattern is not too complicated, that is, it does not contain many fringes or many critical points. So in this case a large $13 \times 13$-pixel neighborhood may be used. This large fitting plane filters out efficiently the noise of the estimated phase.

No fringe analysis system is noise immune; sooner or later a noise energy is reached such that the fringe demodulation system breaks down and gives a useless estimated phase. Of course, the RQPT system is not an exception. So, in the next example (Fig. 4), we have simulated a more complicated noisy fringe pattern with nonconstant modulation $b(x, y)$ and zero background $a(x, y)=0$. The aim of this simulation is to stretch the RQPT robustness to the limit in terms of noise and fringe complication. As occurs with any other fringe pattern demodulation system, the RQPT may demodulate very noisy fringe patterns whenever the fringes are not so complicated, i.e., it may have high noise but few fringes and few critical points. On the contrary, a fringe pattern cannot be too noisy when a more complicated fringe pattern

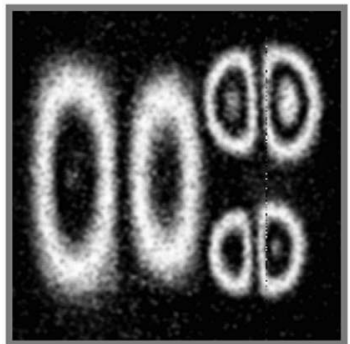

(a)

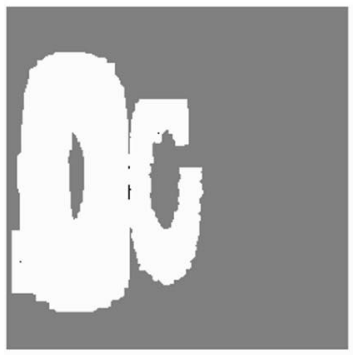

(c)

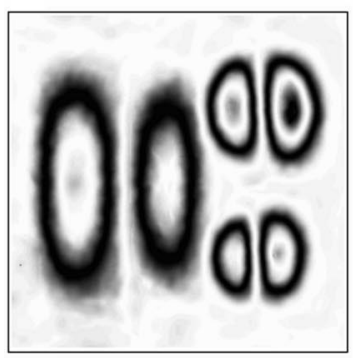

(e)

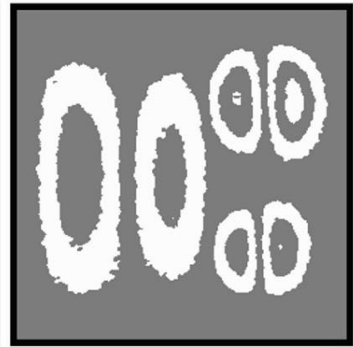

(b)

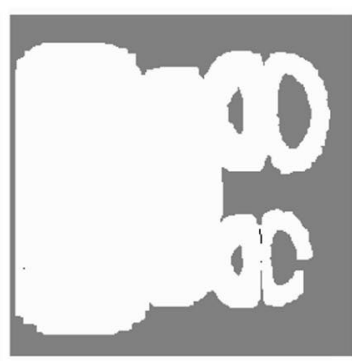

(d)

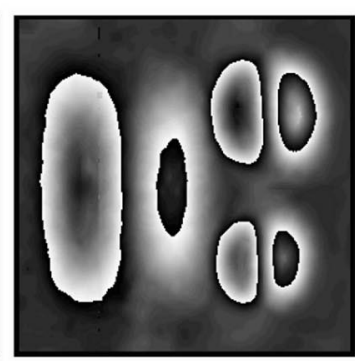

( $f$ )
Fig. 3. Phase demodulation of an experimentally obtained speckle interferometric pattern. (a) Speckle pattern. (b) Traced path that the regularized QPT (RQPT) demodulating system follows. Whiter zones are preferably first demodulated. (c), (d) Two "moments" on the path followed by the RQPT demodulating system. (e) Quadrature of the original fringe pattern. (f) Demodulated phase shown rewrapped.

containing many fringes and many critical points (minima, maxima, or saddles) is analyzed, such as the one shown in Fig. 4(a). Also in Fig. 4 we show some other signals involved in the phase estimation process. Figure 4(b) shows the path along the fringes that the RQPT will follow preferentially. Figure 4(c) shows the quadrature signal obtained by this sequential system. If the noise and/or the fringe pattern is more complicated than that shown in Fig. 4, it is advisable to use as a first step in the demodulation process a robust $2 \mathrm{D}$ filtering and normalizing algorithm before making use of the RQPT.

\section{COMPARISON BETWEEN THE}

\section{REGULARIZED PHASE TRACKER AND THE REGULARIZED QUADRATURE AND PHASE TRACKER ESTIMATORS}

We have analyzed the RQPT system, and we have seen how it can be used to demodulate closed-fringe interferograms. In this section the RQPT system is compared with the RPT system, ${ }^{8}$ and the advantages of the RQPT are pointed out. In the case of demodulating a computergenerated noise-free fringe pattern, both systems give the 
expected continuous and smooth modulating phase, even for a small regularizing neighborhood $N_{x, y}$. The advantage of the RQPT system becomes clearer when a noisy interferogram is analyzed. In this section we show experimentally how the RQPT system is more robust to noise and to fluctuations in the fringe contrast $b(x, y)$ than the RPT system. The reason is that the RQPT system has the additional constraint term related to the quadrature of the data signal.

Let us begin by displaying the local cost functionals for both sequential demodulating systems. For the RPT the local cost functional is

$$
U_{\mathrm{RPT}}=\sum_{(\epsilon, \eta) \in N_{x, y}}\left\{[I-\cos (p)]^{2}+\lambda(\hat{\phi}-p)^{2} m\right\},
$$

and the one corresponding to the RQPT system is

$$
\begin{aligned}
U_{\mathrm{RQPT}}= & \sum_{(\epsilon, \eta) \in N_{x, y}}\left\{[I-\cos (p)]^{2}+\left[I_{x}+\hat{\omega}_{x} \sin (p)\right]^{2}\right. \\
& \left.+\left[I_{y}+\hat{\omega}_{y} \sin (p)\right]^{2}+\lambda(\hat{\phi}-p)^{2} m\right\},
\end{aligned}
$$

where in both cases the regularizing plane is given by

$$
p(\epsilon, \eta)=\hat{\phi}(x)+\hat{\omega}_{x}(x, y)(x-\epsilon)+\hat{\omega}_{x}(x, y)(y-\eta) .
$$

One can see that the difference between these cost functionals is the quadrature-related term, which is $U_{Q}$ $=\left[I_{x}+\hat{\omega}_{x} \sin (p)\right]^{2}+\left[I_{y}+\hat{\omega}_{y} \sin (p)\right]^{2}$. Although it may look like a minor addition, this term has nevertheless important consequences in terms of robustness to noise and rejection of undesired solutions.

As mentioned above, the problem of estimating the modulating phase of a single interferogram containing closed fringes is ill posed, because this problem has infi-

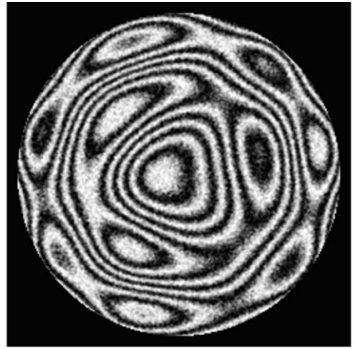

(a)

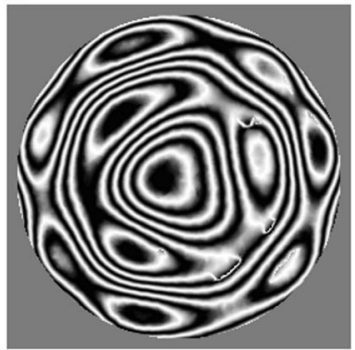

(c)

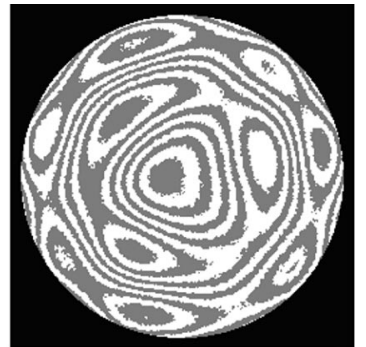

(b)

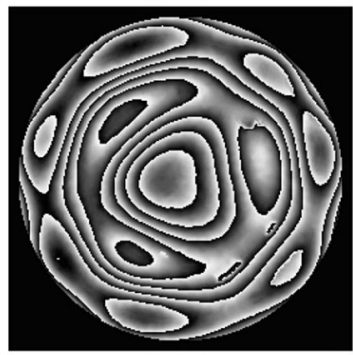

(d)
Fig. 4. Phase demodulation of a noisy computer-generated fringe pattern. (a) Noisy fringe pattern. (b) Traced path that the sequential RQPT demodulating system will follow. Whiter zones are preferably first demodulated. (c) Quadrature of the original fringe pattern. (d) Demodulated phase shown rewrapped. nitely many solutions compatible with the observations. However, the fact that in the minimizing algorithms (RPT or RQPT) one uses the final state of a neighborhood pixel as the initial condition of the pixel being demodulated shrinks the solution space to the set of continuous functions. As a consequence the two main competing continuous solutions that render both local functionals to low values are

$$
\begin{aligned}
& \hat{\phi}_{1}(x) \approx \phi(x, y), \\
& \hat{\phi}_{2}(x) \approx \cos ^{-1}[I(x, y)],
\end{aligned}
$$

where $\cos ^{-1}(\cdot)$ is the inverse of $\cos (\cdot)$ and takes values in the closed interval $[0, \pi]$. These two solutions are continuous functions compatible with the observed data. Of course, the solution given by $\hat{\phi}_{1}(x)$ [Fig. 1(c)] is smoother than that given by $\hat{\phi}_{2}(x)$ [Fig. 1(a)], so the regularizing plane biases the RPT/RQPT systems toward $\hat{\phi}_{1}(x)$. However, in noisy situations, the noisy estimate of $\hat{\phi}_{1}$ may fall too close to $\hat{\phi}_{2}$, and the RPT system may fail to recover the desired solution $\hat{\phi}_{1}(x)$. On the other hand, the RQPT system has two additional constraint terms that add robustness to the system and give the expected minimum $\hat{\phi}_{1}$ in many cases where the RPT fails.

One example of the higher robustness of the RQPT over the RPT system may be seen in a contrast mismatch situation between the fringe model and the fringe data. In Fig. 5(a) we show the following simple noise-free computer-generated fringe pattern:

$$
I(x, y)=0.8 \cos \left(\omega_{0} x\right) .
$$

In this case the RPT system finds the competing solution $\hat{\phi}_{2}$ because the RPT fails to reach close to the phase values of 0 or $\pi$, which would allow it to jump between adjacent Riemann surfaces corresponding to $\cos ^{-1}(\cdot)$ in order to obtain $\omega_{0} x$ instead of obtaining $\hat{\phi}=\cos ^{-1}[I(x, y)]$ [Fig. 5 (b)] as a solution. So in this case the RPT remains on a single branch of the Riemann surface regardless of the size of the neighborhood $N_{x, y}$. On the other hand, the RQPT finds the desired solution $\omega_{0} x$ [Fig. 5(c)] because this system implicitly calculates the quadrature of the fringes. In this numerical experiment (Fig. 5), we have used a neighborhood $N_{x, y}=5$ and the parameter $\lambda=5$ for both the RPT and RQPT systems. Note that if the amplitude's fringe model and the actual fringe amplitude diverge even further in this direction, i.e., $I(x, y)$ $=0.5 \cos \left(\omega_{0} x\right)$, maintaining a normalized fringe model, both systems fail to recover the desired function $\omega_{0} x$.

In the next example we consider the noisy interferogram

$$
I(x, y)=\cos \left[\omega_{0} x+n(x, y)\right] .
$$

Here we have a perfect match between the data and the fringe model's contrast. However, in this case, we have added some phase noise $n(x, y)$. This noise is uniformly distributed in the range $[-1,1]$ rad. Figure $6(\mathrm{a})$ shows the fringe pattern, and Figs. 6(b) and 6(c) show the demodulated phase obtained by the RPT and RQPT systems, respectively. As one can see, the RQPT obtains a good approximation of the desired phase $\omega_{0} x$, while the RPT again obtains the wrong solution $\cos ^{-1}\left[\cos \left(\omega_{0} x\right)\right]$. In 
this numerical experiment we have used a neighborhood $N_{x, y}=9$ and the parameter $\lambda=5$ for both the RPT and RQPT systems.

It should be noted that in Ref. 8 it was proposed that an additional term to the RPT cost functional be introduced, in order to increase the robustness with respect to noise; the resulting cost functional is

$$
\begin{aligned}
U_{\mathrm{RPT}}= & \sum_{(\epsilon, \eta) \in N_{x, y}}\left\{[I-\cos (p)]^{2}+[I-\cos (p+\alpha)]^{2}\right. \\
& \left.+\lambda(\hat{\phi}-p)^{2} m\right\}
\end{aligned}
$$

where $\alpha$ is a piston phase shift (usually $\alpha=\pi / 4$ ) introduced into the fringe model. This is a rather awkward constraint that also forces the phase-shifted model to resemble the original fringe data. Although this trick may permit one to obtain a solution in the correct branch of the Riemann surface in noisy interferograms, it nevertheless distorts the resulting phase estimation. This is because the two terms cannot be made equal to zero simultaneously. So a compromise between them is taken, and this compromise is the expected demodulated signal slightly distorted. In the RPT paper ${ }^{8}$ it is advised that after the distorted phase is obtained, this distortion may be removed by minimizing the unshifted functional (28), taking as initial condition the optimum $\hat{\phi}(x)$ obtained

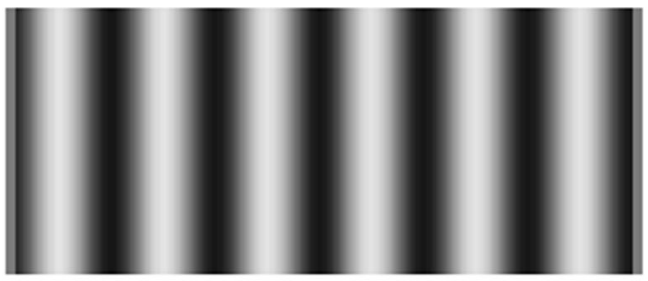

(a)

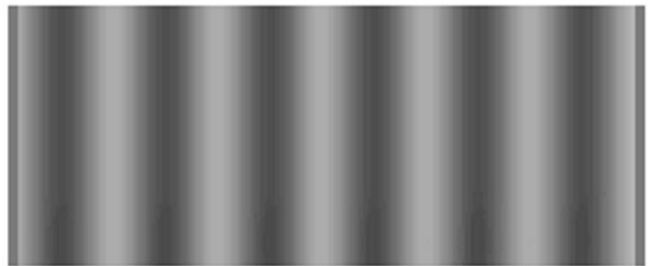

( b )
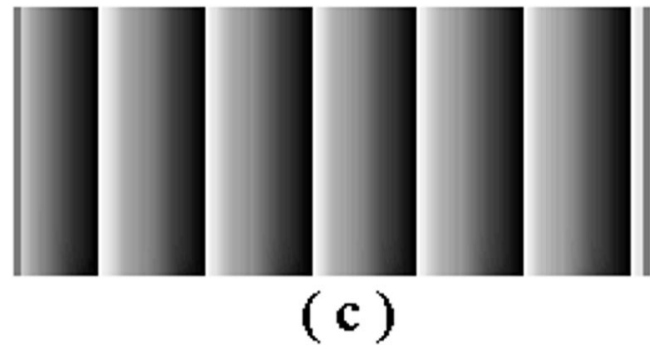

Fig. 5. Phase demodulation of a fringe pattern with less than expected fringe contrast of 1.0. (a) Fringe pattern $I(x)$ $=0.8 \cos \left(\omega_{0} x\right)$. (b) Wrong demodulated phase found by the RPT [Eq. (28)], which is close to $\hat{\phi}(x)=\cos ^{-1}[I(x)]$. (c) Correctly demodulated phase using the RQPT system.

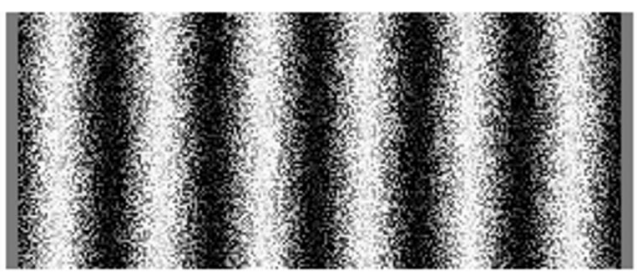

(a)

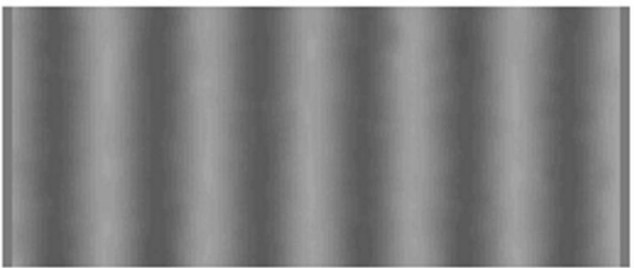

( b )

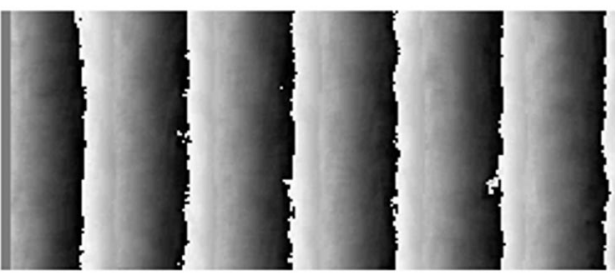

(c)

Fig. 6. Phase demodulation of a noisy fringe pattern with the right (expected) contrast equal to 1.0. (a) Noisy fringe pattern $I(x)=\cos [\phi(x)+\operatorname{noise}(x)]$. (b) Wrong demodulated phase using the RPT system given by Eq. (28), which is close to $\hat{\phi}(x)$ $=\cos ^{-1}[I(x)]$. (c) Correctly demodulated phase obtained by the RQPT system.

from the phase-shifted functional (35). This heuristic trick is not necessary in the RQPT system presented in this work.

\section{CONCLUSIONS}

We have shown that the estimation of the local phase of the fringe pattern may be made more robust and stable if the quadrature of the fringe pattern-which depends on its gradient and local frequency-is estimated at the same time. This estimation process is effective only if the phase variation is locally monotonic, i.e., if the fringes are (locally) open. It is possible, however, to use this scheme to estimate the phase of patterns with closed fringes as well, provided that one scans the image in such a way that the demodulator always sees an open-fringe pattern and has a good initial estimate of its phase and local frequency. This will not be the case, of course, for critical points (maxima, minima, or saddles) of the phase surface, but if these difficult spots are demodulated after the phase has been estimated at all their surrounding pixels, the QPT operator will obtain a correct value there as well. We have also shown that the QPT estimator may be made robust to noise by estimating the parameters of a linear (planar) approximation to the phase surface in a neighborhood, instead of the phase and frequency values at the point, obtaining in this case the 
RQPT. The robustness with respect to noise improves if the size of this neighborhood is increased, but its maximum size is limited by the smoothness of the underlying phase surface, i.e., the size of the larger window where a linear approximation to the phase remains valid.

The RQPT system may have difficulties in demodulating even noise-free fringe patterns having some of their critical points near or at the boundary of the fringe pattern. This is because at these places there are no closed paths around the critical points and the main strategy of surrounding the critical points by following the fringes may fail.

Finally, we have made a detailed comparison of the RQPT system presented here with the previously published RPT demodulator. ${ }^{8}$ We have stressed their similarities and differences and made it clear that the RQPT is the next logical step for the improvement of the RPT.

\section{ACKNOWLEDGMENTS}

M. Servin and J. L. Marroquin were partially supported by grants 33429-E and 42523, respectively, from Consejo Nacional de Ciencia y Tecnología (CONACYT), Mexico.

Manuel Servin Guirado, the corresponding author, may be reached by e-mail at mservin@cio.mx.

\section{REFERENCES}

1. D. Malacara, M. Servin, and Z. Malacara, Interferogram Analysis for Optical Testing (Marcel Dekker, New York, 1998), Chap. 1.
2. M. Takeda, H. Ina, and S. Kobayashi, "Fourier-transform method of fringe pattern analysis," J. Opt. Soc. Am. 72, 156-160 (1982).

3. D. Malacara, M. Servin, and Z. Malacara, Interferogram Analysis for Optical Testing (Marcel Dekker, New York, 1998), Chap. 6.

4. G. Cloud, Optical Methods of Engineering Analysis (Cambridge U. Press, Cambridge, UK, 1995).

5. K. G. Larkin, D. J. Bone, and M. A. Oldfield, "Natural demodulation of two-dimensional fringe patterns. I. General background of the spiral phase quadrature transform," J. Opt. Soc. Am. A 18, 1862-1870 (2001).

6. M. Servin, J. A. Quiroga, and J. L. Marroquin, "General $n$-dimensional quadrature transform and its application to interferogram demodulation,” J. Opt. Soc. Am. A 20, 925934 (2003).

7. J. A. Quiroga, M. Servin, and F. J. Cuevas, "Modulo $2 \pi$ fringe orientation angle estimation by phase unwrapping with a regularized phase tracking algorithm," J. Opt. Soc. Am. A 19, 1524-1531 (2002).

8. M. Servin, J. L. Marroquin, and F. J. Cuevas, "Fringefollower regularized phase tracker for demodulation of closed-fringe interferograms," J. Opt. Soc. Am. A 18, 689695 (2001).

9. M. Servin and R. Rodriguez-Vera, "Two dimensional phase locked loop demodulation of carrier frequency interferograms," J. Mod. Opt. 40, 2087-2094 (1993).

10. J. Kozlowski and G. Serra, "New modified phase locked loop method for fringe pattern demodulation," Opt. Eng. 36, 2025-2030 (1997).

11. J. Kozlowski and G. Serra, "Complex phase tracing method for fringe pattern demodulation," Appl. Opt. 38, 2256-2262 (1999).

12. B. Ströbel, "Processing of interferometric phase maps as complex-valued phasor images," Appl. Opt. 35, 2192-2198 (1996).

13. C. W. Groetsch, Inverse Problems in the Mathematical Sciences (Vieweg, Braunschweig, Germany, 1993). 